\title{
Bifurcations and phase transitions in spatially extended two-member hypercycles
}

\author{
Josep Sardanyés ${ }^{\mathrm{a}}$, Ricard V. Soléa,b,* \\ ${ }^{a}$ Complex Systems Lab (ICREA-UPF), Barcelona Biomedical Research Park ( PRBB-GRIB), Dr. Aiguader 88, 08003 Barcelona, Spain \\ ${ }^{\mathrm{b}}$ Santa Fe Institute, 1399 Hyde Park Road, Santa Fe NM 87501, USA
}

Received 31 August 2005; received in revised form 5 July 2006; accepted 17 July 2006

Available online 21 July 2006

\begin{abstract}
Mounting theoretical and experimental evidence indicates that the success of molecular replicators is strongly tied to the local nature of their interactions. Local dispersal in a given spatial domain, particularly on surfaces, might strongly enhance the growth and selection of fit molecules and their resistance to parasites. In this work the spatial dynamics of a simple hypercycle model consisting of two molecular species is analysed. In order to characterize it, both mean field models and stochastic, spatially explicit approaches are considered. The mean field approach predicts the presence of a saddle-node bifurcation separating a phase involving stable hypercycles from extinction, consistently with spatially explicit models, where an absorbing first-order phase transition is shown to exist and diffusion is explicitly introduced. The saddle-node bifurcation is shown to leave a ghost in the phase plane. A metapopulation-based model is also developed in order to account for the observed phases when both diffusion and reaction are considered. The role of information and diffusion as well as the relevance of these phases and the underlying spatial structures are discussed, and their potential implications for the evolution of early replicators are outlined.
\end{abstract}

(C) 2006 Elsevier Ltd. All rights reserved.

Keywords: Hypercycles; Spatial replicator dynamics; Metapopulations; Bifurcations; Phase transitions

\section{Introduction}

The hypercycle (Eigen and Schuster, 1979) is a catalytic network composed of self-replicating units, which are able to catalyse the replication of one other unit, altogether forming a closed loop (see Fig. 1) (Eigen and Schuster, 1979; Szathmáry and Maynard Smith, 1997). This cyclically coupled array of self-replicative species has been considered as a possible molecular network of prebiotic replicators in the context of the origins of life and has also been suggested as an important step in the transition from inanimate to living chemistry (Lee et al., 1997a). Nowadays, one naturally occurring hypercycle has been proved in a real system. Specifically, Eigen et al. (1991) showed the hypercyclic, i.e. autocatalytic, organization in a short growth phase of a coliphage DNA and its replicase machinery. The important selective and evolutionary

\footnotetext{
${ }^{*}$ Corresponding author. Tel.: + 349331 60532; fax: + 34932213237 .

E-mail address: ricard.sole@upf.edu (R.V. Solé).
}

properties of the hypercycle make such networks good candidates to explain key steps in prebiotic evolution. For instance, the entire hypercyclic population is able to compete more efficiently for existing resources than any one member on its own, and beneficial mutations of any one member are spread over the whole system of replicators (Lee et al., 1997b). Moreover, hypercyclic organization allows the cooperative selection of competing replicators, ensuring the stability of large contents of information, not possible in non-catalytically coupled replicators, i.e. quasi-species (Eigen and Schuster, 1979; Nuño et al., 1993b).

It is well known that the hypercyclic organization allows to avoid the so-called informational crisis caused by the error catastrophe (Eigen, 2002; Eigen and Schuster, 1979; García-Tejedor et al., 1988; Solé and Goodwin, 2000; Stadler and Stadler, 2003; Szathmáry and Maynard Smith, 1997). Essentially, for a given mutation rate $\mu$, error-prone replicators have a sharp limit to the information they can carry that can be maintained by natural selection (Swetina 


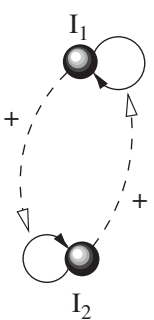

(a)

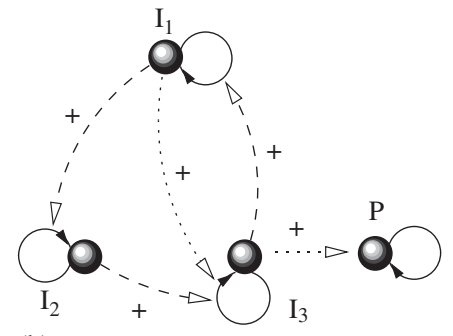

(b)
Fig. 1. Simple hypercycles: (a) two-member and (b) three-member catalytic networks. Catalytic interactions are represented with dashed arrows, curved solid arrows show replicator self-replicating activity. Hypercycles can become unstable due to so-called shortcuts or as a consequence of parasites, $P$, shown in (b) and indicated with dotted arrows.

and Schuster, 1982). In other words, if the sequence length of a replicator is $v$, then it has an upper limit to genome complexity given by $v_{c} \sim \mu^{-1}$. This actually means that genome length is not allowed to increase beyond $v_{c}$ unless mechanisms of error correction are present. The hypercycle is able to avoid the error threshold transition: each component keeps itself under the critical size individually but the information of the whole system overcomes such a limitation.

Although early models of these molecular assemblies ignored spatial constraints in an explicit way, space has been shown to be a key component. Specifically, the local nature of interactions that takes place in reality (where systems are far from well mixed) pervade the emergence of new types of phenomena, from spatial structures to complex dynamics (Bascompte and Solé, 1998). This is the case at all scales, from complex ecosystems (Bascompte and Solé, 1995; Solé and Bascompte, 2006) to molecular ecology (Breyer et al., 1998; Füchslin and McCaskill, 2001; McCaskill, 1997). Space plays a key role in hypercycle dynamics, and spatial models of hypercyclic organization have been explored (Boerlijst, 2000; Chacón and Moran, 1993; Cronhjort, 1995; Cronhjort and Blomberg, 1994; Fontanari and Ferreira, 2002; Scheuring et al., 2002; Szabó et al., 2002). In this context, prebiotic chemical evolution could have begun on a surface-bonded autocatalytic chemical network (anionically bonded to positively charged mineral surfaces like pyrite) (Fontanari and Ferreira, 2002; Smith and Szathmáry, 2001; Wächtershäuser, 1988). Surface-bonded chemical networks have kinetic and thermodynamic advantages because thermodynamic equilibrium in a surface favors synthesis, not degradation as would occur in solution (Wächtershäuser, 1988) (see also Scheuring et al., 2002).

In surface-bonded systems, unlikely molecular encounters among hypercyclic replicators would be needed to generate new replicators able to maintain the stability of the hypercycle, so reactants should be strongly bonded to the surface but they should also be able to migrate laterally on it. Previous studies on hypercycles have shown a rich diversity of behavioral patterns, both in mean field (Nuño, 1993a,b; Rowe, 1997; Stadler and Stadler, 2003) and spatial dynamics (Boerlijst and Hogeweg, 1991; Cronhjort, 2000; Cronhjort and Blomberg, 1994, 1997; Czarán and Szathmáry, 2000; Fontanari and Ferreira, 2002; GarcíaTejedor et al., 1988). The simplest hypercycle is made up of only two catalytic members (Fig. 1a) and is likely to be the earlier example of these cooperative entities that might have emerged. The two-member hypercycle has been experimentally achieved with two self-replicating peptides sharing one common fragment (Lee et al., 1997b). Theoretical studies with such a hypercycle, i.e. twomember, have been mainly focused on the role of the error-prone replication in the stability of this network (Nuño et al., 1993a,b).

In order to fully characterize the possible domains of coexistence and extinction, here we analyse the twomember hypercycle from several theoretical approaches, emphasizing in the role of space in its dynamics. Mean field and cellular automata models are developed to gain insight into the expected overall behavior, as well as into the stochastic and discrete dynamics of such a spatially extended hypercycle, respectively. At this level of analysis, each component of the hypercycle is limited to a molecule lacking any well-defined sequence. Two basic reactions are considered when space is not taken into account: reaction and degradation. The first one represents the second-order, cross-catalytic reaction, which involves two possible events:

$I_{1}+I_{2}+s \stackrel{k}{\longrightarrow} 2 I_{1}+I_{2}$,

$I_{1}+I_{2}+s \stackrel{\eta_{k} k}{\longrightarrow} I_{1}+2 I_{2}$,

where $k$ is the reaction rate. Here the two members of the hypercycle react and the catalytic interaction generates a new component from available monomers (labeled $s$ ). Note that we represent the general case considering $\eta_{k}$. This constant (with $\eta_{k} \neq 1$ ) indicates a different self-replication rate among both replicators because of a different informational content. Reactions (1) and (2) unfold hyperbolic growth (Eigen and Schuster, 1979; Szathmáry and Maynard Smith, 1997) that reaches infinite species concentration in finite time, so we need to define a population constraint in order to impose a dilution flux (although it is also possible to implement a reaction of hydrolysis to limit the growth of replicators, Fontanari and Ferreira, 2002). The second basic reaction represents molecular decay due to hydrolysis, according to

$I_{1} \stackrel{\varepsilon}{\longrightarrow} S$,

$I_{2} \stackrel{\eta_{\varepsilon} \varepsilon}{\longrightarrow} S$,

$\varepsilon$ being the decay rate. Here we also use a constant, $\eta_{\varepsilon}$, indicating that the second replicator might have different decay properties. Such differences would be the result of different information for both hypercycle species.

It has been suggested that the dynamics of a catalytic network is, at least in part, determined by its graph 
structure (Stadler and Stadler, 2003). Hypercycles are stable against dynamical perturbations but they are unstable against structural perturbations of the network. The structural perturbations that may lead to dynamical instabilities in hypercycles are given by "shortcuts" and parasites (e.g. selfish replicators) (Cronhjort and Blomberg, 1997; Eigen and Schuster, 1979; Stadler and Stadler, 2003). We underline that the two-member hypercycle can act as a switch making such "shortcuts" in larger hypercycles. In Fig. $1 \mathrm{~b}$ we show a possible catalytic change causing such a short-cut.

\section{Non-spatial dynamics}

The first step in our analysis is to summarize the qualitative features displayed by the two-member hypercycle without spatial degrees of freedom. This case thus corresponds to a well-mixed population of hypercycles. A simple mean field model for such a system can be defined in terms of two coupled differential equations, given by the dynamical system:

$\frac{\mathrm{d} x_{1}}{\mathrm{~d} t}=\Phi_{\mu}^{(1)}(\vec{x})=k \Psi\left(x_{1}, x_{2}\right)-\varepsilon x_{1}$,

$\frac{\mathrm{d} x_{2}}{\mathrm{~d} t}=\Phi_{\mu}^{(2)}(\vec{x})=\eta_{k} k \Psi\left(x_{1}, x_{2}\right)-\eta_{\varepsilon} \varepsilon x_{2}$.

Here $x_{1,2}$ is the concentration of molecule $I_{1,2} ; k$ and $\varepsilon$ represent chemical formation and decay rates, respectively. Constants $\eta_{k}$ and $\eta_{\varepsilon}$ indicate, as previously mentioned, the degree of assymetry among both molecules, which might contain different genetic information responsible for different kinetic properties. Obviously, the case $\eta_{k}=\eta_{\varepsilon}=$ 1 would represent the symmetric hypercycle, in which both species are indistinguishable. The coupling between the two species is given by the function

$\Psi(\vec{x})=x_{1} x_{2}\left(1-\frac{x_{1}+x_{2}}{c_{0}}\right)$,

$c_{0}$ being the carrying capacity (hereafter $c_{0}=1$ ).

The properties of the previous model are easily determined by using linear stability analysis of the fixed points. It is not difficult to see that three fixed points are present. These include the trivial one $\left(x_{1}^{*}=0, x_{2}^{*}=0\right)$, and two additional points $\left(\left(\eta_{\varepsilon} / \eta_{k}\right) \Gamma_{ \pm}, \Gamma_{ \pm}\right)$, with

$\Gamma_{ \pm}=\frac{1}{2 \xi}\left[1 \pm \sqrt{1-\frac{4 \varepsilon \xi}{k}}\right]$,

here with $\xi \equiv 1+\eta_{\varepsilon} / \eta_{k}$. For the simplest non-trivial case given by the symmetric two-member hypercycle, $\xi=2$, and thus these fixed points become

$x_{ \pm}^{*(1)}=x_{ \pm}^{*(2)}=\frac{1}{4}\left[1 \pm \sqrt{1-\frac{8 \varepsilon}{k}}\right]$.
Note that both fixed points $\left(\left(\eta_{\varepsilon} / \eta_{k}\right) \Gamma_{ \pm}, \Gamma_{ \pm}\right)$do not exist if $\varepsilon>k / 4 \xi$, so above the threshold, i.e. bifurcation point, $\varepsilon_{c}=$ $k / 4 \xi$ only the trivial fixed point exists in phase space. Such a critical decay rate for the symmetric case is $\varepsilon_{c}=k / 8$. The stability of the three fixed points is determined from the eigenvalues of the Jacobi matrix

$L_{\mu}=\left(\begin{array}{ll}\frac{\partial \Phi_{\mu}^{(1)}(\vec{x})}{\partial x_{1}} & \frac{\partial \Phi_{\mu}^{(1)}(\vec{x})}{\partial x_{2}} \\ \frac{\partial \Phi_{\mu}^{(2)}(\vec{x})}{\partial x_{1}} & \frac{\partial \Phi_{\mu}^{(2)}(\vec{x})}{\partial x_{2}}\end{array}\right)$.

For our system, it is given

$L_{\mu}=\left(\begin{array}{cc}f_{1}-\varepsilon & f_{2} \\ \eta_{k} f_{1} & \eta_{k} f_{2}-\eta_{\varepsilon} \varepsilon\end{array}\right)$,

where $f_{i}=k x_{j}\left(1-S-x_{i}\right) \quad$ with $\quad(i, j=1,2 ; i \neq j) \quad$ and $\left(S \equiv x_{1}+x_{2}\right)$. Now from the eigenvalues we determine the stability of the fixed points. For the fixed point $(0,0)$, we have

$L_{\mu}(\mathbf{0})=\left(\begin{array}{cc}-\varepsilon-\lambda & 0 \\ 0 & -\eta_{\varepsilon} \varepsilon-\lambda\end{array}\right)$,

which gives two negative eigenvalues i.e. $\lambda_{1}=-\varepsilon$ and $\lambda_{2}=-\eta_{\varepsilon} \varepsilon$, so this point is negative and thus always stable, representing the extinction attractor in phase space. Here an initial condition starting in the close surrounding of such a point will drive to the extinction of both replicators: a minimum initial concentration is needed in order to maintain this hypercycle. For the other two fixed points $\left(\boldsymbol{\Gamma}_{ \pm}\right)$, we have

$L_{\mu}\left(\Gamma_{ \pm}, \Gamma_{ \pm}\right)=\left(\begin{array}{cc}\alpha-\varepsilon & \alpha \\ \eta_{k} \alpha & \eta_{k} \alpha-\eta_{\varepsilon} \varepsilon\end{array}\right)$,

with $\alpha \equiv k \Gamma_{ \pm}-\left(1+2\left(\eta_{\varepsilon} / \eta_{k}\right)\right) k \Gamma_{ \pm}^{2}$. If $\eta_{\varepsilon}=\eta_{k}=1$ (the symmetric case), we have $\alpha=k \Gamma_{ \pm}-3 k \Gamma_{ \pm}^{2}$. Here the eigenvalues are given by $\lambda_{-}\left(\boldsymbol{\Gamma}_{ \pm}\right)=-\varepsilon$, and $\lambda_{+}\left(\boldsymbol{\Gamma}_{ \pm}\right)=$ $2 \alpha-\varepsilon$, with

$\lambda_{+}\left(\Gamma_{ \pm}\right)=-\frac{k}{4}\left(1+\sqrt{1-\frac{8 \varepsilon}{k}}\right)+2 \varepsilon$,

$\lambda_{+}\left(\boldsymbol{\Gamma}_{+}\right)$being negative and $\lambda_{+}\left(\boldsymbol{\Gamma}_{-}\right)$positive, indicating that both fixed points $\left(\boldsymbol{\Gamma}_{+}\right)$and $\left(\boldsymbol{\Gamma}_{-}\right)$are, respectively, a stable node and a saddle. The stability of the fixed points for the asymmetric hypercycle is, as shown below, equivalent to the invariant states for the symmetric case.

The qualitative behavior exhibited by the hypercycle is summarized in Fig. 2, where phase portraits obtained solving Eqs. (5)-(6) numerically are represented. For the symmetric case (i.e. $\eta_{k}=\eta_{\varepsilon}=1$ ), and with $\varepsilon<\varepsilon_{c}$ (Figs. 2a and b), trajectories flow toward both stable fixed points, depending on the initial condition. The saddle-node is found in between these stable fixed points. Here, the 
symmetric hypercycle
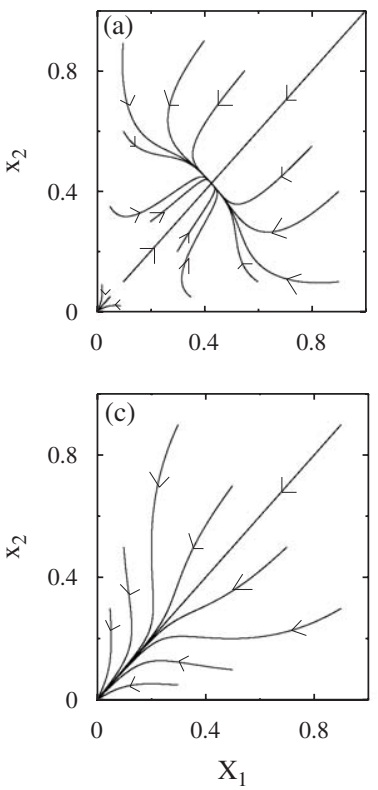
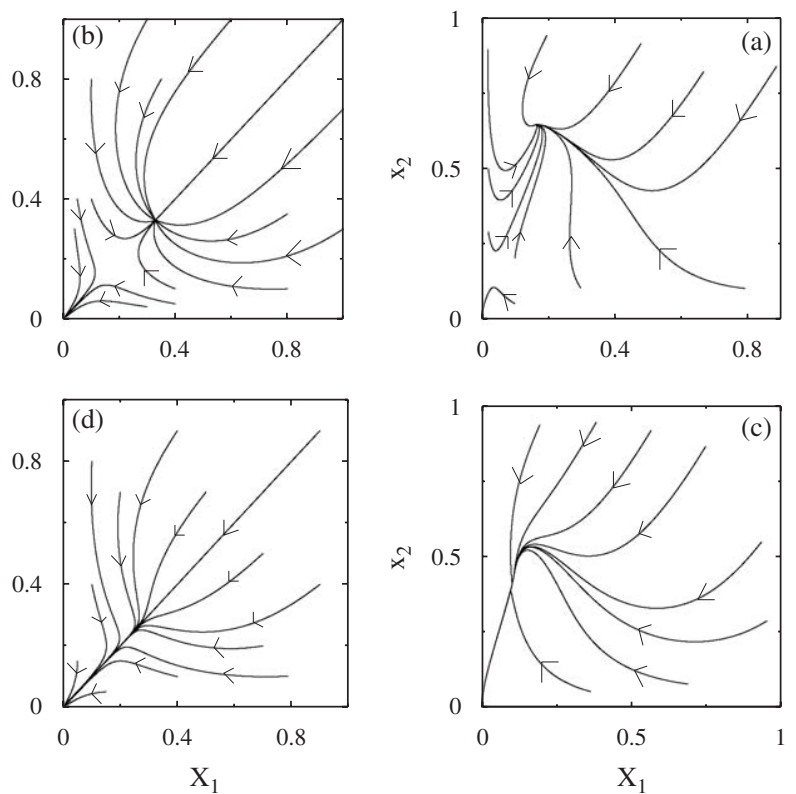

asymmetric hypercycle

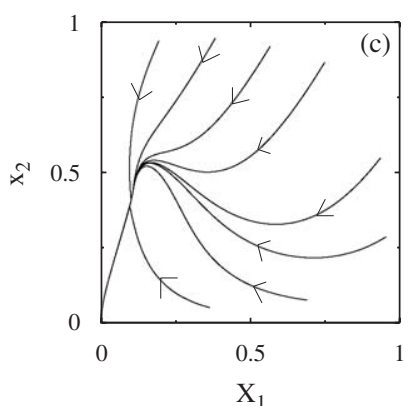

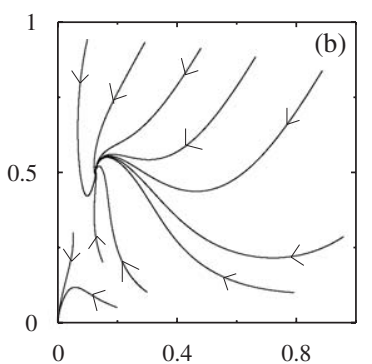

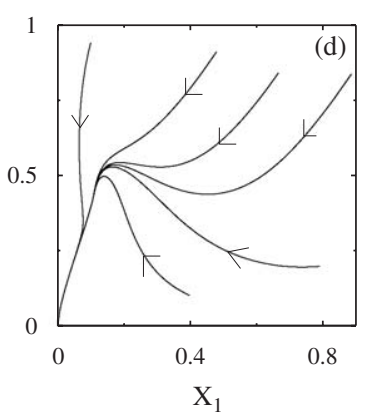

Fig. 2. Phase portraits for both symmetric and asymmetric hypercycles. For the symmetric case: (a) $\varepsilon=0.05$ and $k=0.8$; (b) $\varepsilon=0.09$ and $k=0.8$; (c) $\varepsilon=0.06$ and $k=0.4$; and (d) $\varepsilon=0.13$ and $k=1$. And for the asymmetric case: $k=0.8, \eta_{k}=2, \eta_{\varepsilon}=0.5$ and (a) $\varepsilon=0.1$, (b) $\varepsilon=0.15$, (c) $\varepsilon=0.16005$, and (d) $\varepsilon=0.161$. In both cases and from (a) to (d), the saddle-node and the coexistence node approach each other until a saddle-node bifurcation occurs, then both fixed points coalesce and all the flow is sucked into the extinction attractor. The bifurcation leaves a remnant saddle, i.e. ghost, in the phase plane. The arrows indicate the direction of the flow.

coexistence node has a wide basin of attraction and low concentration values for $I_{1}$ and $I_{2}$ as initial conditions fall into the extinction fixed point $\left(x_{1}^{*}=0, x_{2}^{*}=0\right)$. Note that the increase in the decay rate involves the motion of the coexistence stable node toward the saddle-node, reducing its basin of attraction, while the extinction basin of attraction increases. When the decay rate exceeds the bifurcation point all the flow in phase space move toward the extinction point $\left(x_{1}^{*}=0, x_{2}^{*}=0\right)$, which becomes globally stable due to a saddle-node bifurcation (Figs. 2c and $\mathrm{d}$ with $\varepsilon>\varepsilon_{c}$ ). The flows for the asymmetric hypercycle also show the same qualitative behavior. Note that the asymmetry does not change the stability of the fixed points. Below the bifurcation point we find a stable coexistence point and the stable extinction attractor, which are separated by the saddle. Here the second species achieves higher asymptotic concentration values for the coexistence fixed point because its self-replication and decay rates are, respectively, higher and lower (see Fig. 2 for details).

It is possible that two fixed points that have annihilated each other continue, by means of the so-called ghost, influencing the flow sucking trajectories and delaying them before allowing the movement to the other fixed point (Strogatz, 2000; Strogatz and Westervelt, 1989). The "apparition" of a saddle-node ghost is shown in the dynamics of Eqs. (5)-(6) (see Figs. 2c, d). Some trajectories flow and are exponentially damped to a region placed far away from the attractor point $\left(x_{1}^{*}=0, x_{2}^{*}=0\right)$ before flowing to such a point. In Fig. 3 we show the bifurcation diagram for both symmetric and asymmetric hypercycles.

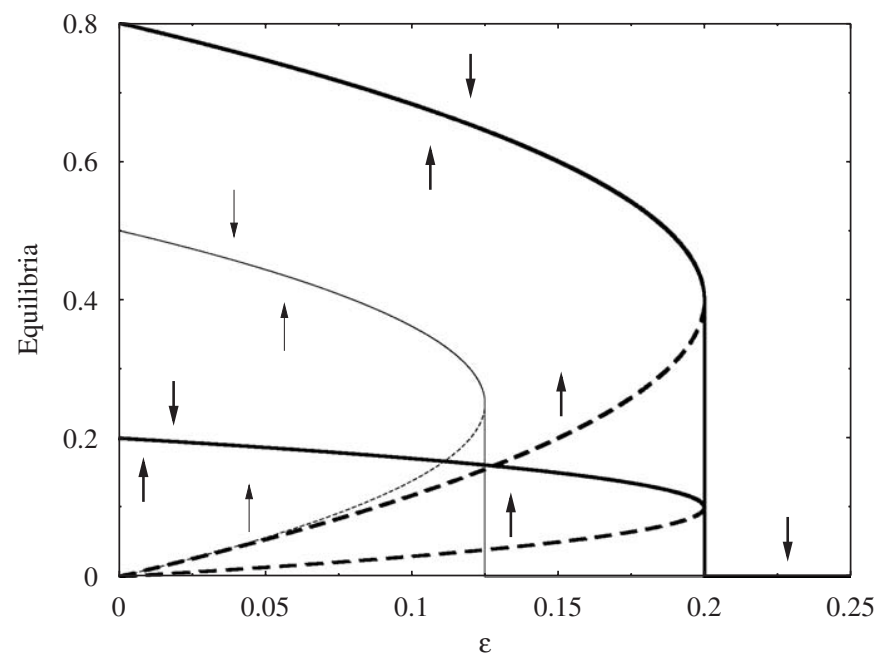

Fig. 3. Bifurcation diagram for the symmetric (thin lines) and asymmetric (thick lines with $\eta_{k}=2$ and $\eta_{\varepsilon}=0.5$ ) two-member hypercycle, by using the decay rate, $\varepsilon$, as control parameter with $k=1$. The vertical axis indicates the equilibria for the fixed points of the hypercycle. In the asymmetric case, each member of the hypercycle has a different invariant concentration value. Here the higher values are achieved by the second member of the network. The solid lines indicate the stable equilibria given, for the symmetric hypercycle, by the stable fixed point $\left(\boldsymbol{\Gamma}_{+}\right)$and by $\left(\left(\eta_{\varepsilon} / \eta_{k}\right) \Gamma_{+}, \Gamma_{+}\right)$ for the asymmetric one. Dashed lines correspond to the non-stable point according to $\left(\boldsymbol{\Gamma}_{-}\right)$and to $\left(\left(\eta_{\varepsilon} / \eta_{k}\right) \Gamma_{-}, \Gamma_{-}\right)$. As $\varepsilon$ increases, both equilibria approach until $\varepsilon_{c}$, beyond which a saddle-node bifurcation takes place.

Here the crossing of the bifurcation point, $\varepsilon_{c} \approx k / 4 \xi$, involves the coalescence of the coexistence stable node and the saddle. 


\section{Spatial models}

In this section we analyse the dynamics of the twomember hypercycle by considering spatial degrees of freedom. In our study we differentiate between two types of diffusion. The first one is the diffusion of new replicators, which happens after the interaction between both members of the hypercycle. Here such an interaction generates a new replicator that can, at rate $\delta$, stick on a neighboring cell. However, this new molecule can also leave the system with probability $(1-\delta)$, because of external stochastic fluctuations. The other type of diffusion is associated with a random and local movement of the hypercyclic replicators through the spatial domain.

Firstly, a general, one-dimensional mean field model considering diffusion $\delta$ is used in Section 3.1. In Section 3.2 we also explore either the symmetric and asymmetric networks now considering space explicitly, using stochastic cellular automata (CA) models (Ilachinsky, 2002). Specifically, we study the dynamics of such a hypercycle extended in both one-dimensional and two-dimensional spaces by defining appropriate microscopic rules.

\subsection{Mean field model}

Let us first introduce a simple mean field model describing the dynamics of an asymmetric two-member hypercycle and considering diffusion linked to replication $(\delta)$, which can be represented with the next one-dimensional discrete equation:

$\rho_{t+1}=\rho_{t}+R\left[\rho_{t} ; \eta_{k}, k, \delta, q\right]-E\left[\rho_{t} ; \eta_{\varepsilon}, \varepsilon\right]$,

where $\rho_{t}$ is the overall density of replicators at a given time step $t$. The term $R$ in the right-hand side includes all the events associated with reaction dynamics, where $k, \delta$ and $q$ are the reaction rate, diffusion due to replication and the number of nearest neighbors, respectively. On the other hand, the term $E$ introduces a molecular decay because of spontaneous hydrolysis, $\varepsilon$ being the decay rate. We take $\zeta=k \delta q$ as the effective reaction rate. A continuous approximation to Eq. (15) can be obtained according to

$\rho_{t+1}-\rho_{t} \approx \frac{\mathrm{d} \rho\left((R, \mu), E\left(\mu^{\prime}\right)\right)}{\mathrm{d} t}$,

$\mu$ and $\mu^{\prime}$ being the sets of parameters for both reaction and decay terms, respectively. Here $\dot{\rho}\left(R(\mu), E\left(\mu^{\prime}\right)\right)$ is derived from Eqs. (5) and (6). From such equations, now with $\rho_{1} \equiv$ $x_{1}$ and $\rho_{2} \equiv x_{2}$, we can obtain a closed approximate expression for the total population $\rho \equiv \rho_{1}+\rho_{2}$, thus having

$\frac{\mathrm{d} \rho}{\mathrm{d} t}=k\left(1+\eta_{k}\right)(1-\rho) \rho_{1} \rho_{2}-\varepsilon\left(\rho_{1}+\eta_{\varepsilon} \rho_{2}\right)$.

Assuming that the system $\left(\rho_{1}, \rho_{2}\right)$ is close to the direction defined by the line

$\rho_{1}=\frac{\eta_{\varepsilon}}{\eta_{k}} \rho_{2}$, which corresponds to the one defined by the eigenvector associated with the fixed point $\left(\left(\eta_{\varepsilon} / \eta_{k}\right) \Gamma_{+}, \Gamma_{+}\right)$described before, using (18) and $\rho \equiv \rho_{1}+\rho_{2}$, we can show that

$\rho_{1}=\rho-\frac{\rho}{\Theta}$

and

$\rho_{2}=\frac{\rho}{\Theta}$,

where $\Theta \equiv\left(\eta_{\varepsilon} / \eta_{k}\right)+1$. The resulting one-dimensional model, including the effective reaction rate, $\zeta$, is given by

$\frac{\mathrm{d} \rho}{\mathrm{d} t}=\zeta\left(1+\eta_{k}\right)(1-\rho) \frac{\rho}{\Theta} \Lambda-\varepsilon\left[\Lambda+\eta_{\varepsilon} \frac{\rho}{\Theta}\right]$,

with

$\Lambda=\left(\rho-\frac{\rho}{\Theta}\right)$.

The set of equilibrium points for Eq. (21) is given by $\Pi_{\mu}=\left\{\rho^{*} \in \mathbb{R}^{+} \mid\left(0, \rho_{+}^{*}, \rho_{-}^{*}\right)\right\}$, with

$\rho_{ \pm}^{*}=\frac{1}{2}[1 \pm \sqrt{1-4 \Omega}]$,

where

$\Omega=\frac{\varepsilon \omega \Theta^{2}}{\zeta\left(1+\eta_{k}\right)(\Theta-1)}$

and

$\omega=1-\frac{1}{\Theta}+\eta_{\varepsilon} \frac{1}{\Theta}$.

From the fixed points $\rho_{ \pm}^{*}$ the critical decay rate, i.e. bifurcation point, is defined to be

$\varepsilon_{c}=\frac{\zeta\left(1+\eta_{k}\right)(\Theta-1)}{4 \omega \Theta^{2}}$.

Such a bifurcation point for the symmetric hypercycle (with $\eta_{k}=\eta_{\varepsilon}=1, \quad \Theta=2$ and $\omega=1$ ) is given by $\varepsilon_{c}=k \delta q / 8$.

Let us now analyse the stability of the fixed points of Eq. (21) for the symmetric case, in which the dynamical system is given by

$\frac{\mathrm{d} \rho}{\mathrm{d} t}=2 \zeta\left(\frac{\rho}{2}\right)^{2}(1-\rho)-\varepsilon \rho$,

where the reaction term is according to $2 \zeta(\rho / 2)^{2}(1-\rho)$. This term indicates that, at a rate $\zeta$, empty sites (here with probability $(1-\rho))$ are occupied provided two different (but indistinguishable) molecules meet together (with probability $\left.(\rho / 2)^{2}\right)$. Thus Eq. (24) can be written as

$\frac{\mathrm{d} \rho}{\mathrm{d} t}=f_{\mu}=\rho^{2}(1-\rho)-\frac{2 \varepsilon}{\zeta} \rho$.

Using linear stability analysis we have

$\lambda_{\mu}=\frac{\mathrm{d} f_{\mu}}{\mathrm{d} \rho}=\rho(2-3 \rho)-\alpha$,

where $\alpha=2 \varepsilon / \zeta$. It is easy to show that for $\lambda_{\mu}\left(\rho^{*}=0\right)=-\alpha$, $\lambda_{\mu}<0$, thus the fixed point ( 0 ) is always stable. We now 
analyse the stability of the non-trivial fixed points $\left\{\rho_{+}^{*}, \rho_{-}^{*}\right\}$. For both points we have

$\lambda_{\mu}\left(\rho_{ \pm}^{*}\right)=\frac{1}{2}(1 \pm \beta)\left(2-\frac{3}{2}(1 \pm \beta)\right)-\alpha$.

Here $\beta=\sqrt{1-4 \alpha}$. It can be shown that below the critical condition i.e. $\alpha<\frac{1}{4}, \lambda_{\mu}\left(\rho_{+}^{*}\right)<0$ and $\lambda_{\mu}\left(\rho_{-}^{*}\right)>0$, so both fixed points are stable and unstable, respectively.

The bifurcation scenario of this one-dimensional model is shown in Fig. 4A. We specifically represent four different scenarios for the symmetric hypercycle with increasing values of $\delta$, and for two different values of $q$. Here, it is shown that the increase in both diffusion and the number of neighbors involves a higher critical decay rate, becoming the hypercycle more resistant to hydrolysis. In this diagram we also show two cases corresponding to the asymmetric network (with thick lines and $\eta_{k}=2$ and $\eta_{\varepsilon}=0.5$ ), which are shown to have a higher critical decay value than the symmetric case with the same parameters. Here the bifurcation point is also displaced to higher values at increasing diffusion $\delta$ (see Fig. 4A for details).

In Fig. 4B we show the time evolution of the total population for two different diffusion values and for the cases corresponding to $q=4$ and 8 . Here, as previously mentioned, it is shown that diffusion as well as the spatial availability during replication (i.e. number of neighbors) provides stability to the hypercycle. Such a stability is increased by the asymmetric network, which is shown to achieve higher stationary concentration values (see Fig. 4B for details). We finally represent in Figs. $4 \mathrm{C}$ and D, the parameter space plotting $\varepsilon$ and $k$ against $\delta$ for both symmetric and asymmetric hypercycles. Note that in the asymmetric networks the extinction region is reduced. In the $(k, \delta)$ space a hyperbolic critical line separates the two basic phases involving coexistence and extinction.

\subsection{Stochastic cellular automata}

A first approach to the dynamics of the spatially extended two-member hypercycle is obtained by developing a stochastic one-dimensional CA. The two-dimensional implementation on a surface is also considered for both symmetric and asymmetric networks. The spatial analyses for the asymmetric hypercycle allows to focus on the effect of both diffusion and information for these spatially extended hypercycles. The approaches for the symmetric case can also be applied to characterize the spatial dynamics of a single autocatalytic replicator. The state transition rules used in both CAs are implemented as follows:

1. Catalytic self-replication (Fig. 5(ii)): If the cell contains both hypercycle members, one of them (randomly chosen) will replicate, with reaction probability $k$ (for $I_{1}$ ) or with $\eta_{k} k \in(0,1)$ (for $\left.I_{2}\right)$ and diffuse, with probability $\delta$, to a neighboring site only if such a neighbor does not contain the same replicator species.
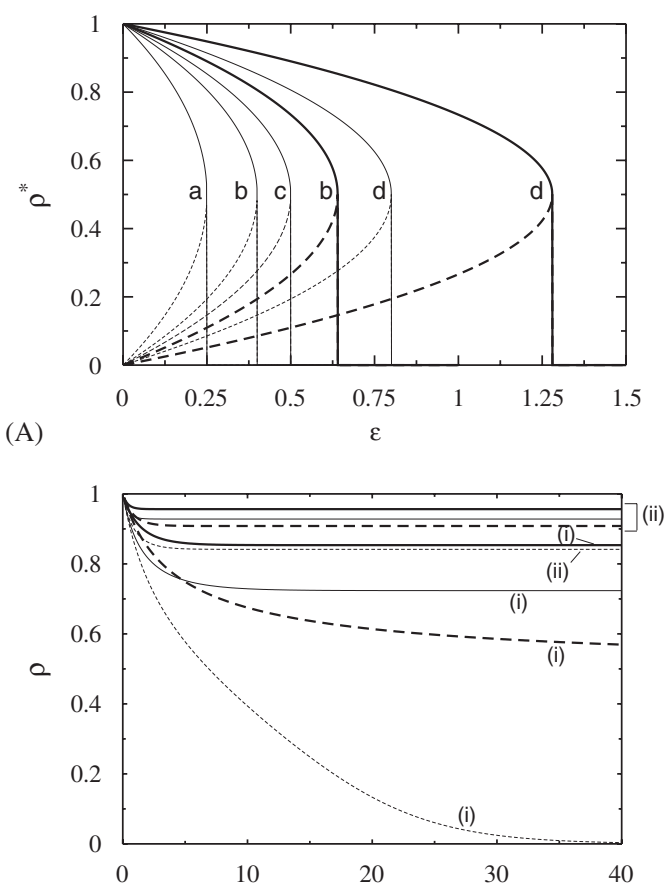

(B)

Fig. 4. (A) Equilibria values for the one-dimensional mean field model Eq. (21) with $k=1$, given by the stable point $\rho_{+}^{*}$ (solid line), and by the unstable one $\rho_{-}^{*}$ (dashed line). Here (a) $\delta=0.5$ and $q=4$; (b) $\delta=0.5$ and $q=8$; (c) $\delta=0.8$ and $q=4$; and (d) $\delta=0.8$ and $q=8$. Fine and thick lines correspond, respectively, to the symmetric and asymmetric hypercycle (with $\eta_{k}=2$ and $\eta_{\varepsilon}=0.5$ ). (B) Time series for the total population with $k=1, \varepsilon=0.2$ and (i) $\delta=0.25$; (ii) $\delta=0.75$. Here fine and thick lines also indicate, respectively, the symmetric and asymmetric networks, and dashed and solid ones indicate $q=4$ and $q=8$, respectively. In (C) and (D) we display two parameter spaces corresponding to both symmetric and asymmetric hypercycles. Note that here the asymmetry reduces the extinction region in both parameter spaces. 


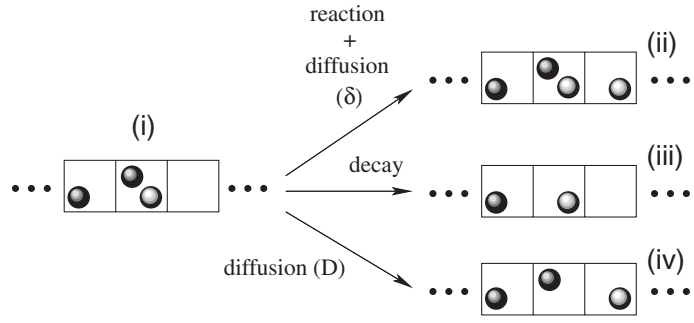

(a) (i)

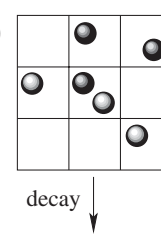

(iii)

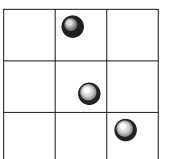

(b)

reaction

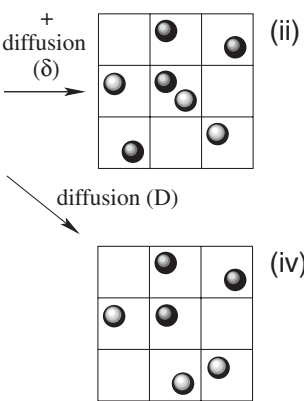

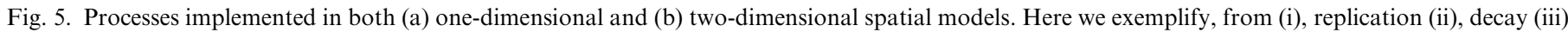

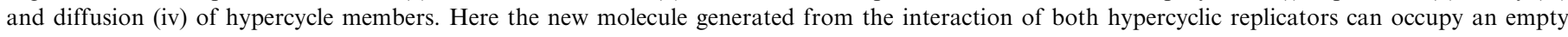

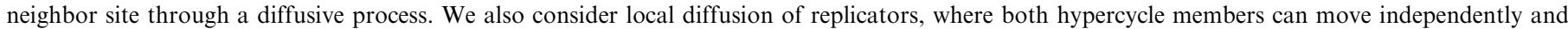
randomly to neighboring cells.
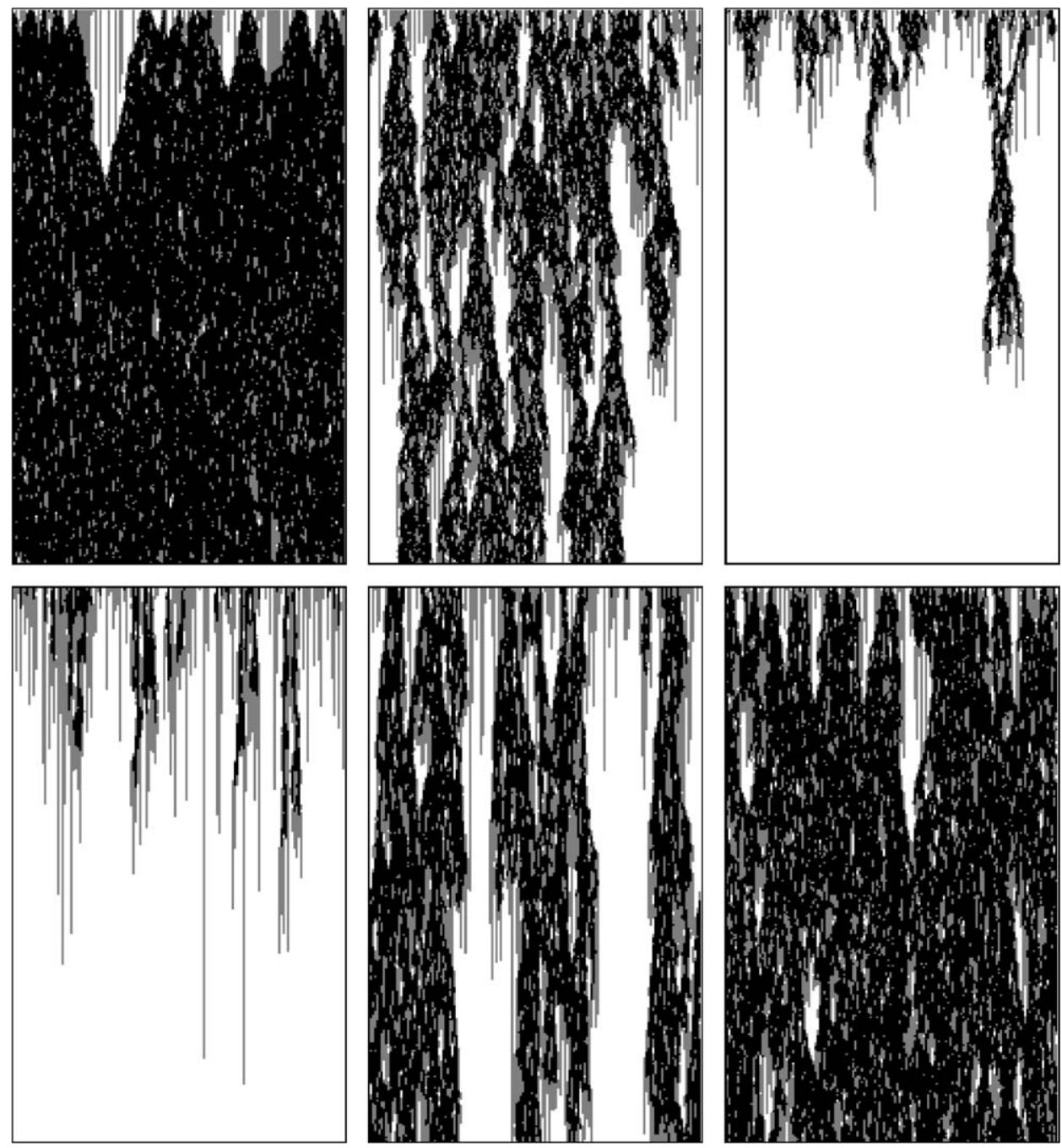

Fig. 6. Space-time diagram in the one-dimensional cellular automaton for the symmetric (i.e. $\eta_{k}=\eta_{\varepsilon}=1$ ) two-member hypercycle. Space goes left-right and time goes top-down. Here three possible states are shown: empty cells (white), cells occupied by a single molecule (gray) or by both members of the hypercycle (black), respectively. The first row shows three different runs with $k=1, \delta=1$ and (from left to right): $\varepsilon=0.02, \varepsilon=0.06$ and $\varepsilon=0.09$. The second row represents the evolution of both hypercyclic replicators with $k=1, \varepsilon=0.03$ and (from left to right): $\delta=0.25, \delta=0.5$ and $\delta=0.75$.

2. Molecular decay (Fig. 5(iii)): The first and the second hypercycle members decay, respectively, with probabilities $\varepsilon$ and $\eta_{\varepsilon} \varepsilon \in(0,1)$, leaving empty space in the cells.
3. Local diffusion (Fig. 5 (iv)): $I_{1}$ and $I_{2}$ move independently to random neighbors with diffusion probability $D_{1}$ and $D_{2}$, respectively, provided the neighbor site does 
not contain the diffusing replicator. We assume that $D_{1}=D_{2} \equiv D$, thus the asymmetry of the hypercycle does not involve different diffusion properties.

In the one-dimensional CA the state space is a row of $L$ cells with periodic boundary conditions. We assume that all cells contain the molecular building blocks, $s$, necessary for the synthesis of new replicators. Transition rules 1 and 2 are applied synchronously while 3 is applied asynchronously, taking $L$ cells at random at each generation. Here, initial conditions are set by inoculating both hypercycle members at random. Our computational approach allows to study the space-time evolution only considering diffusion $\delta$ (i.e. $D=0$ ). Both extinction and persistence phases, previously characterized, are shown in Fig. 6 for the symmetric hypercycle. Here (first row) the extinction phase is achieved by increasing decay probabilities. It is also shown (second row) that diffusion $\delta$ provides a source of stability to the hypercycle. Fig. 7 also shows the space-time diagram, now considering diffusion $D$ (with $\delta=1$ ). Here the random movement of replicators plays a positive role allowing the persistence of both replicators. Hence, the phase change (from extinction to persistence) can be achieved by increasing $D$.

The study of the asymmetric hypercycle in the onedimensional space also reveals the positive effect of both diffusion processes (see Fig. 8 for details). The presence of a critical decay rate responsible for the jump between coexistence to extinction phases allows to interpret such a change as a standard absorbing-state phase transition. This is a class of nonequilibrium transition in which the system crosses from an active to an absorbing phase, by the variation of a control parameter. Once the absorbing phase is achieved, the system remains in such a phase forever, with no possibility to escape (Marro and Dickman, 1999).

The relevant interest in surface-bonded chemical dynamics (Wächtershäuser, 1988) has been an important issue of research in prebiotic evolution (Cronhjort and Blomberg, 1994, 1997; Fontanari and Ferreira, 2002; Scheuring et al., 2002). Hence, the previous observations are extended to a surface by using a two-dimensional CA. The automaton rules now work on a $L \times L$ lattice with periodic boundary conditions. Here we use a Moore neighborhood (i.e. eight nearest neighbors) and as initial conditions both hypercycle members are randomly inoculated over the lattice. This automaton is asynchronous: at each generation, $\tau$, we choose $L \times L$ random cells to ensure that on average each site is updated once per generation. The above-mentioned described state-transition rules are applied to these cells.

The computational explorations reveal two different phases, as predicted by our previous mean field analysis: either $I_{1}$ and $I_{2}$ stable populations or extinction. The change among phases is governed by a first-order phase transition. Fig. 9 shows this phase transition for the symmetric case, by plotting the normalized population of $I_{1}$ replicators at increasing $\varepsilon$ probabilities. The critical

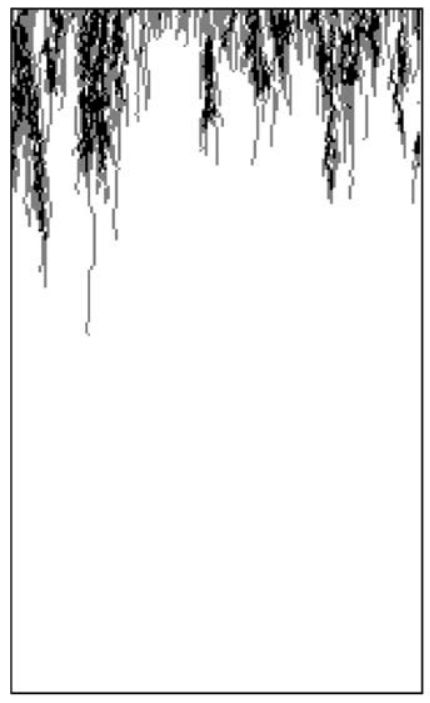

(a)

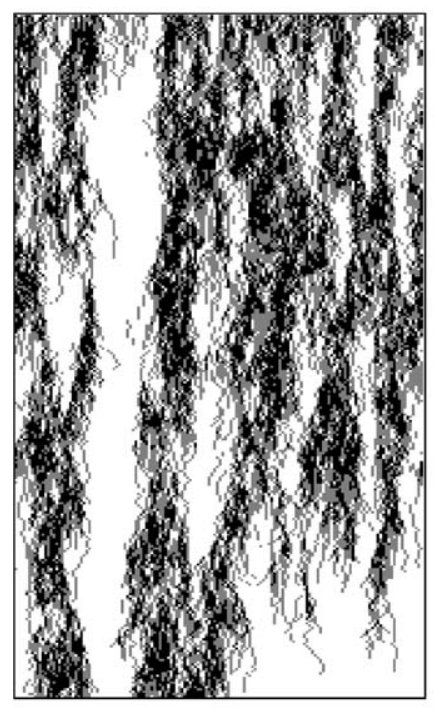

(c)

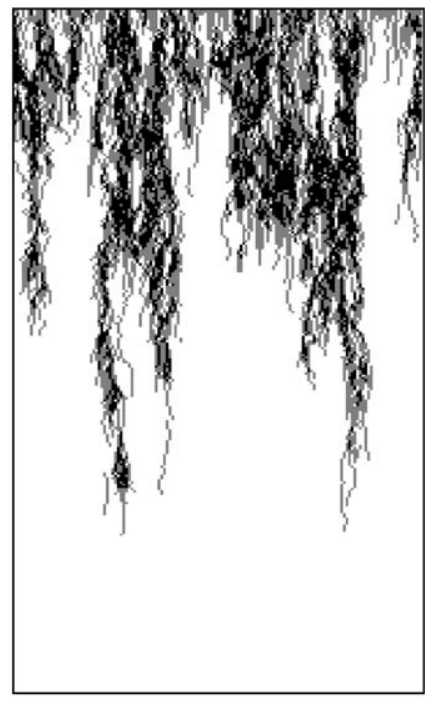

(b)

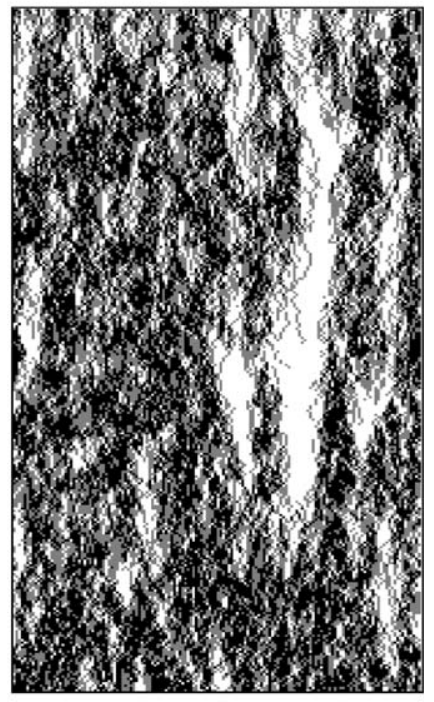

(d)
Fig. 7. Same as Fig. 6 , now with $k=0.8, \delta=1, \varepsilon=0.08$ and with (a) $D=0.1$, (b) $D=0.3$, (c) $D=0.5$, (d) $D=0.7$. By increasing diffusion, the hypercycle is able to achieve persistence.

decay probability with $k=1, \delta=1$ and $D=0$ is found at $\varepsilon_{c} \approx 0.12$. In the inset we also show the parameter space built up by plotting $I_{1}$ density against $k$ and $\delta$. Here the movement of the new replicator molecules caused by the interaction of the hypercyclic members plays a positive role on hypercycle persistence. The numerical results fit the qualitative prediction shown in Fig. 4, with a hyperbolic relation between $\delta$ and $k$. In Fig. 10 we also show the phase transition now considering diffusion $D$. Here the local movement of replicators displaces the critical decay probability to $\varepsilon_{c} \approx 0.15$, showing that diffusion $D$ provides stability to the hypercycle, as we had pointed out in the one-dimensional CA. The inset shows the corresponding time series above (Figs. 10a-c) and below (Figs. 10d-f) $\varepsilon_{c}$. It is clearly shown that below $\varepsilon_{c}$, increasing $\varepsilon$ probabilities decrease the stationary populational levels. 

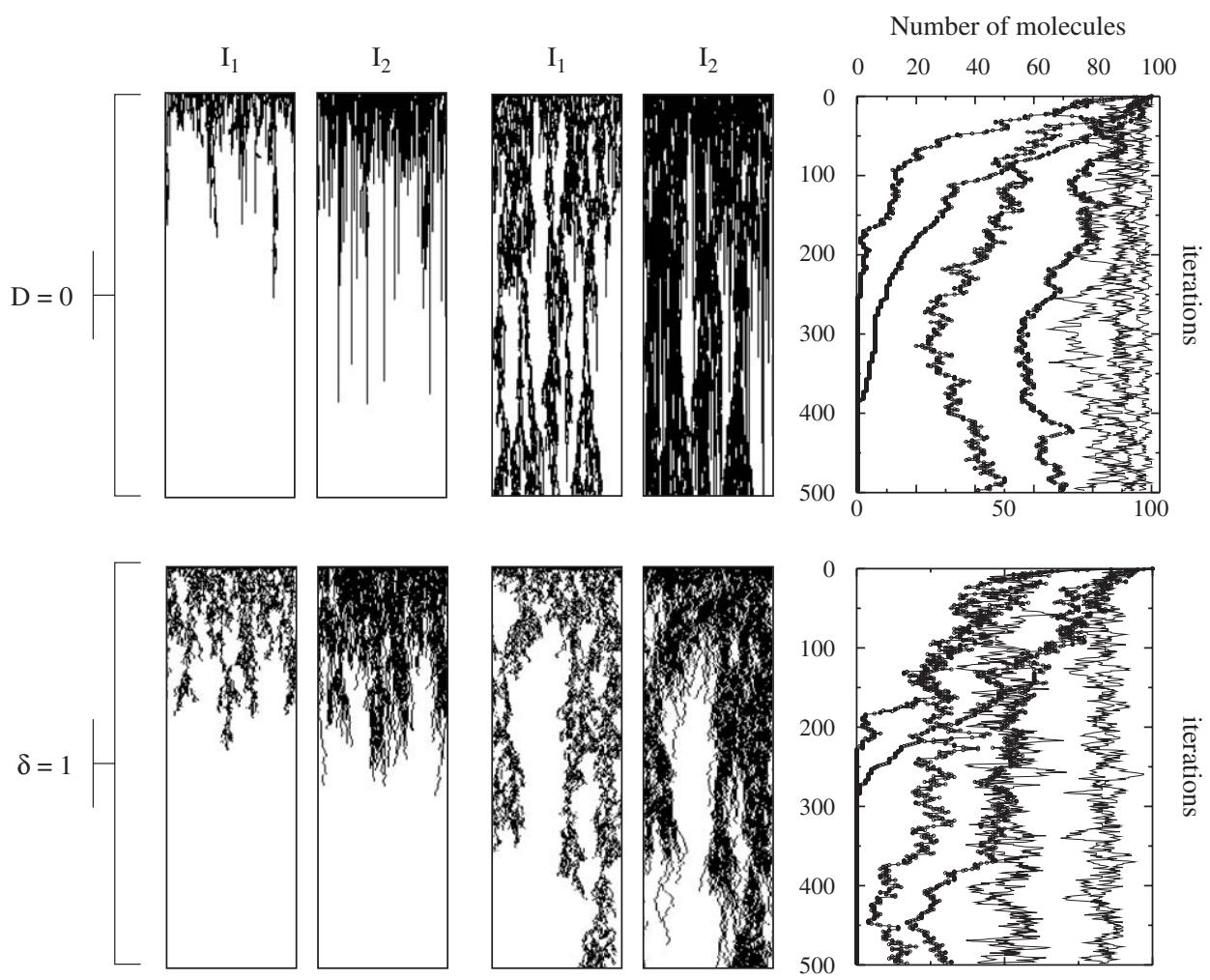

Fig. 8. Spatiotemporal dynamics for the asymmetric hypercycle in the one-dimensional space with $k=1, \eta_{k}=1, \eta_{\varepsilon}=\frac{1}{4}$ and $L=100$. We show two different cases with $D=0$ (up) and $\delta=1$ (down); here as initial conditions all cells are inoculated with both species. In the upper case, the space-time diagrams correspond to both hypercycle members with $\delta=0.2$ (left) and $\delta=0.35$ (right). In the right-hand side we show the time evolution for these two cases (from left to right: first four trajectories with dotted lines) and for two more runs with $\delta=0.6$ and $\delta=1$, represented with thin lines (indicating higher density values). We also show the same results now fixing $\delta=1$, and tunning lateral diffusion. Here the space-time diagrams are obtained for both species with $D=0.2$ (left) and $D=0.5$ (right). The time series also shows these two cases (from left to right: first four trajectories with dotted lines), and two more runs with $D=0.75$ and $D=1$ (thin lines). The increase of both $\delta$ and $D$ involves the persistence of the hypercycle.

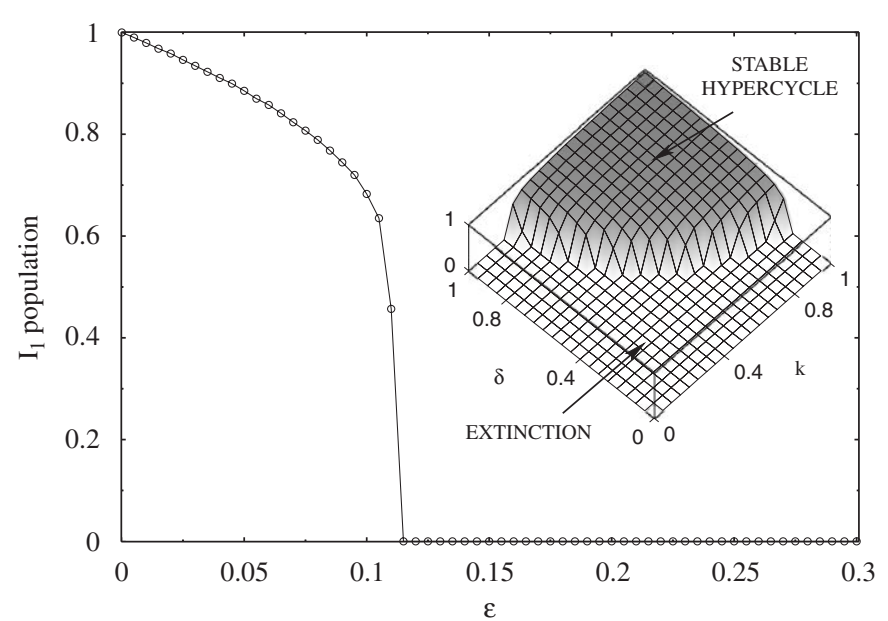

Fig. 9. First-order phase transition in the stochastic, symmetric twomember hypercycle extended on a surface. Main plot shows density values at increasing $\varepsilon$ probabilities without considering lateral diffusion (i.e. $D=0)$ and with $k=1, \delta=1$ and $L=128$. Each data point is the mean taken over 10 replicas of the population value at the end of simulations run over $\tau=5 \times 10^{3}$ generations. The inset shows $I_{1}$ densities for different $k$ and $\delta$ probabilities also for the symmetric network, with $\varepsilon=0.025$ (here $\delta$ is the probability of occupying the neighboring cell when replication takes place).

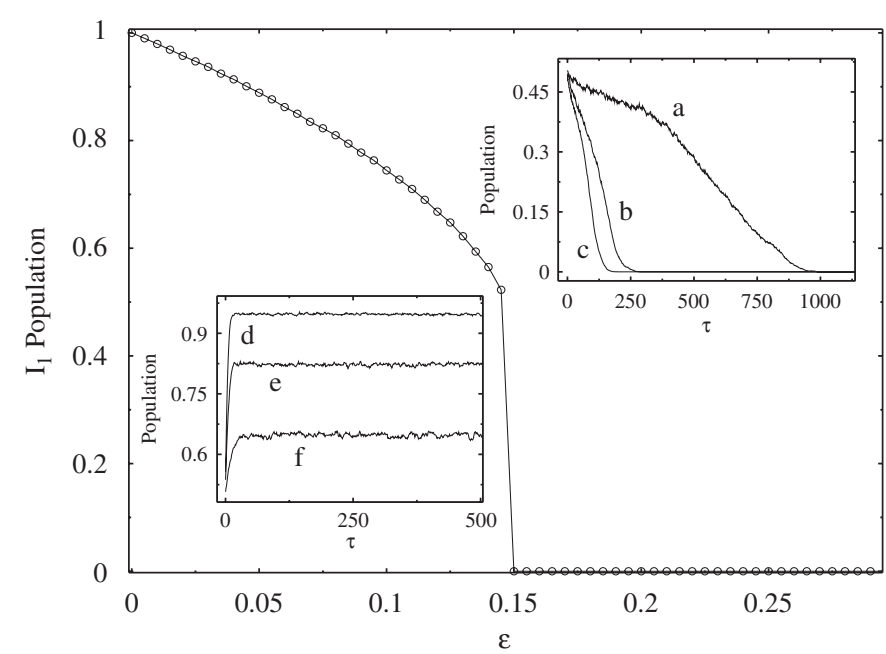

Fig. 10. Same as Fig. 9 now with $D=1$. Here the critical point for the symmetric hypercycle is at $\varepsilon_{c} \approx 0.15$. Beyond such a point the hypercycle is unable to persist. The insets show the time evolution for $I_{1}$ above (upright) and below (left-down) the critical decay: (a) $\varepsilon=0.15$, (b) $\varepsilon=0.155$, (c) $\varepsilon=0.16$; and (d) $\varepsilon=0.025$, (e) $\varepsilon=0.075$ and (f) $\varepsilon=0.125$.

In Fig. 11 we show the spatial patterns for the symmetric network associated with the extinction phase with $k=1$, $\delta=1$ and $\varepsilon=0.1479$. The time evolution for both 


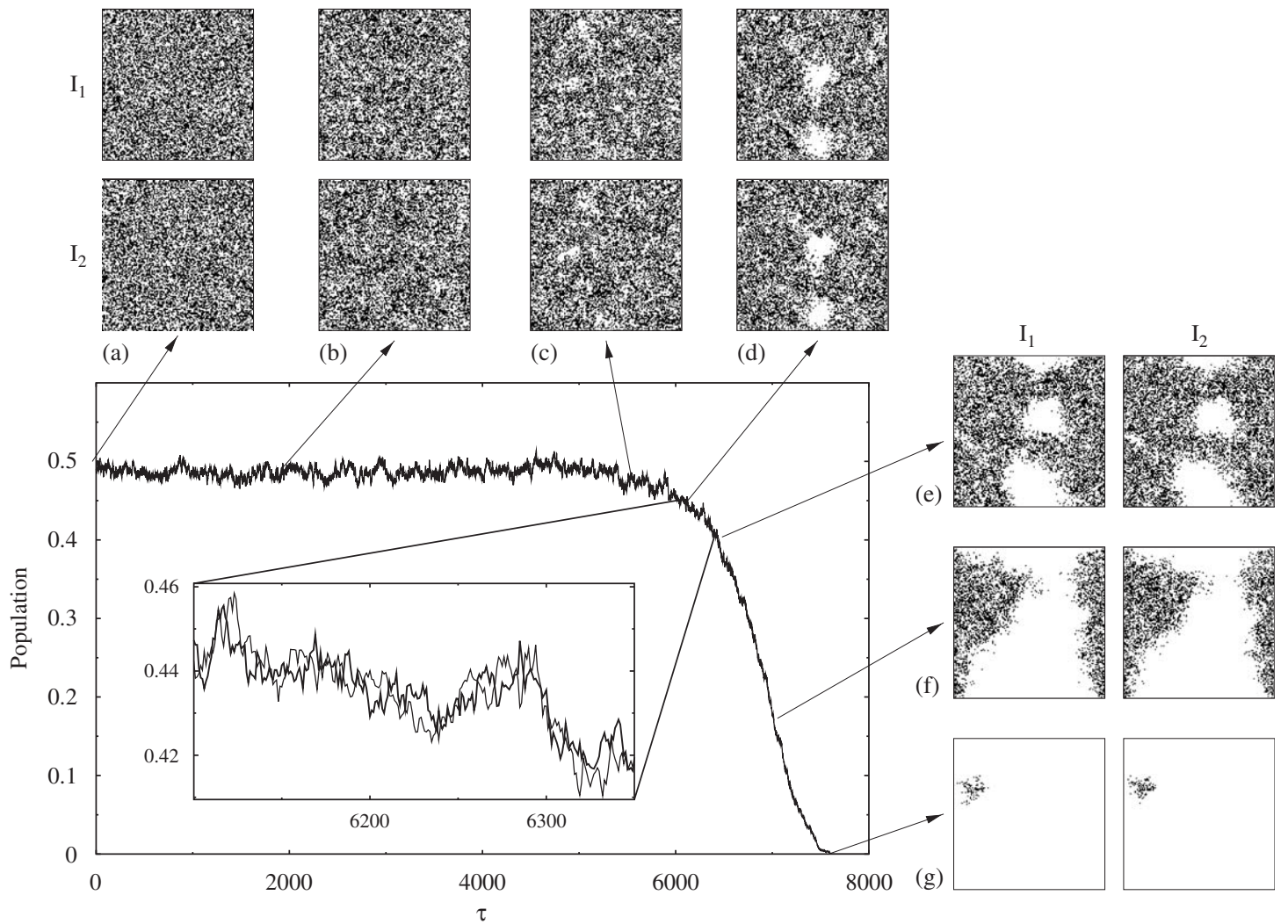

Fig. 11. Spatiotemporal evolution of both hypercycle members in the two-dimensional stochastic CA (here extended on a lattice with $L=128$ ) with $k=1$, $\delta=1, D=1, \varepsilon=0.1479$. Main time series represents $I_{1}$ time evolution and inside the plot we show an enlarged view with both hypercycle members $\left(I_{1}\right.$ : thick line and $I_{2}$ : thin line) time evolution. Arrows indicate $I_{1}$ and $I_{2}$ spatial patterns at (a) the beginning of the simulation, and at (from (b) to (g)) $\tau=2000, \tau=5500, \tau=6000, \tau=6500, \tau=7000$ and $\tau=7500$ generations, respectively. The extinction phase is associated with the presence of an absorbing state.
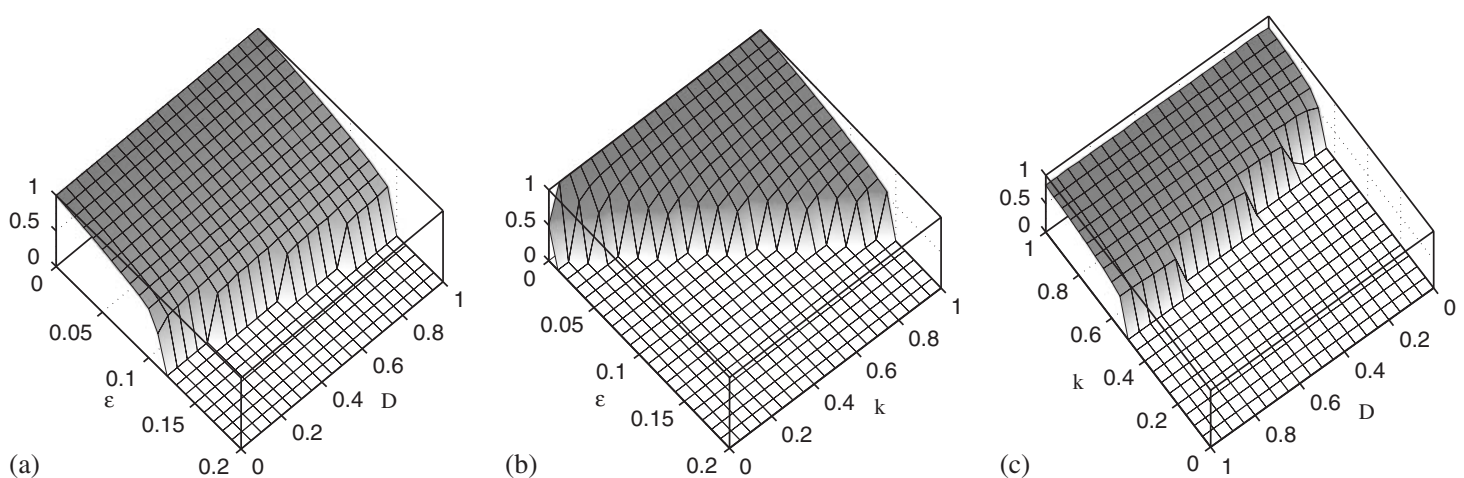

Fig. 12. Population of $I_{1}$ replicators (z-axis) with $\delta=1$ after $\tau=5 \times 10^{3}$ generations on lattices of size $L=128$ : (a) decay-diffusion space with $k=1$, (b) decay-reaction space with $D=1$ and (c) reaction-diffusion space with $\varepsilon=0.075$. Diffusion of replicators $(D)$ through the lattice provides the two-member hypercycle with stability.

hypercycle species is characterized by a first transient phase of small random fluctuations before the extinction of the hypercycle. Fig. 11 also shows $I_{1}$ time series (main plot) and an enlarged view with the time dynamics of both hypercycle members. Note that such an evolution is highly coupled, with both members of the hypercycle displaying quasi-identical occupied regions. The absorbing state is characterized by the presence of empty spots that grow driving the system toward extinction. The role of the parameters involved in this CA is displayed in Fig. 12, where the two phases of the hypercycle are found. As previously mentioned, the diffusion of replicators $(D)$ provides the hypercycle with stability, as shown in Figs. 12a and c.

Up to now we have analysed the interplay between reaction and diffusion starting our simulations with a random distribution of replicators. However, it is interesting to see how diffusion affects the asymptotic dynamics of the hypercyclic members when considering low densities of replicator molecules as initial conditions (as mentioned in 


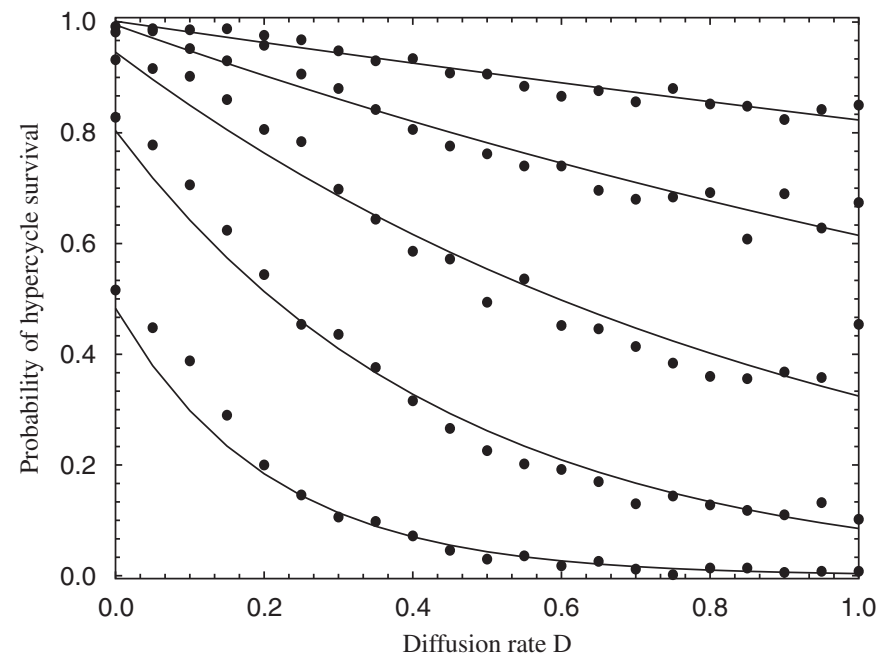

Fig. 13. Hypercycle survival probability with $\delta=1, k=1, \varepsilon=0.075$ and $L=32$, as a function of diffusion probability, $D$. We show five cases with five different and increasing initial conditions with (from down to top) 2, 4, 6, 8 and 10 cells inoculated with both replicators $I_{1}$ and $I_{2}$. Data points represent, in each case, the percentage of runs (averaging over 500 replicas) able to survive and self-maintain in time (considering $I_{1}$ replicator density after $5 \times 10^{3}$ time steps).

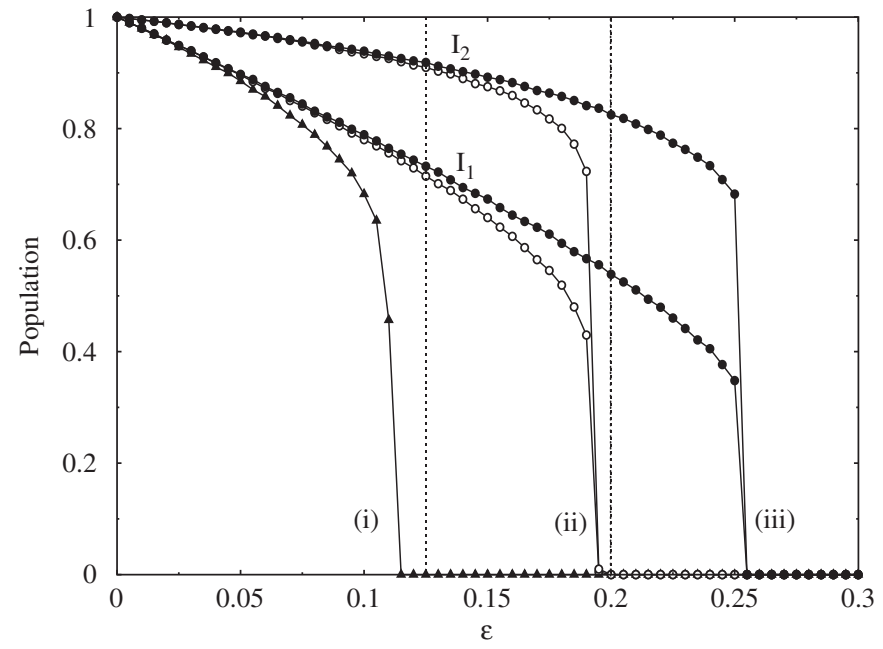

Fig. 14. Same as Fig. 9 with $\delta=1$ and $k=1$. Three different cases are shown: (i) as a reference we display the mean equilibria population values for $I_{1}$ for the symmetric case with $D=0$ (triangles); (ii) the asymmetric hypercycle also with $D=0$ (white circles) and (iii) with $D=1$ (black circles), both with $\eta_{k}=1$ and $\eta_{\varepsilon}=\frac{1}{4}$. Note that with maximum diffusion the critical decay rate is higher, thus the system becomes more resistant to hydrolysis. Vertical dotted lines indicate the critical decay values predicted by the mean field model of Section 2 for the same parameter values used in the simulations for both symmetric (left) and asymmetric networks (right).

Section 2, a minimum initial condition is needed to maintain the hypercycle). In order to explore this point we analyse the behavior of the symmetric system in the two-dimensional stochastic cellular automaton. In Fig. 13 we show the hypercycle survival probability as a function of diffusion (here considered as control parameter) by considering five low-population initial conditions: we inoculate, in the middle of a lattice of size $L=32$, small blobs of replicators $I_{1}$ and $I_{2}$. A monotonous decay is found, as seen from the exponential fitting used as orientation (continuous lines). Note that for very low densities, diffusion plays a negative role in survival. However, this effect decreases as we increase the initial densities, where the hyperbolic growth of the replicators allows high survival probabilities (see Fig. 13 for details).

In Fig. 14 we represent the first-order phase transition and the critical extinction point obtained for the asymmetric hypercycle. As shown for the symmetric case, lateral diffusion plays an important role. Here the critical decay rate without diffusion is placed at $\varepsilon_{c}(D=0) \approx 0.2$; however, with maximum diffusion probability such a value is $\varepsilon_{c}(D=1) \approx 0.25$ (see Fig. 14 for details). Note that the population equilibria of the fittest species, i.e. $I_{2}$, is placed above the equilibria for the first hypercycle species. The dynamics of both survival and extinction phases for the asymmetric network are shown in Fig. 15. In the

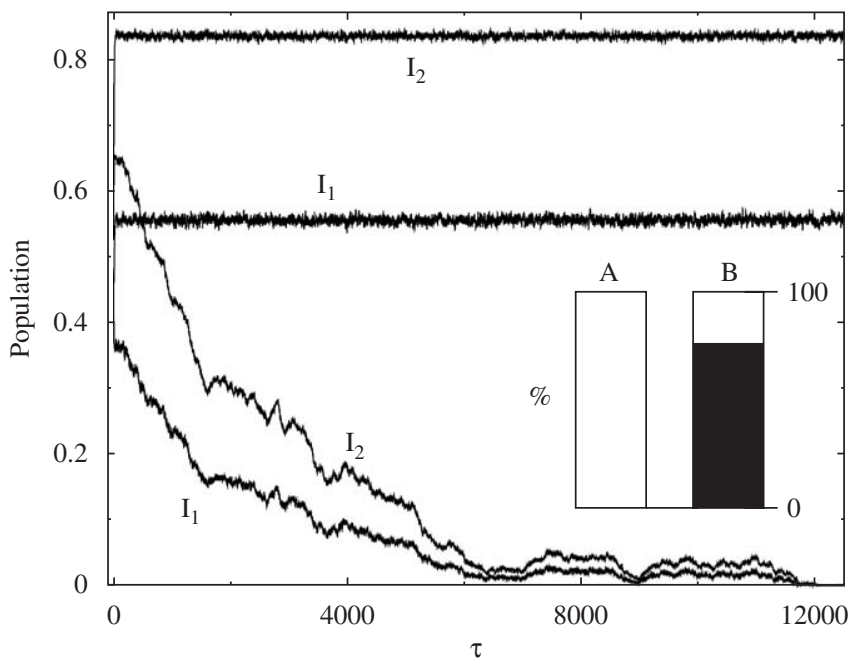

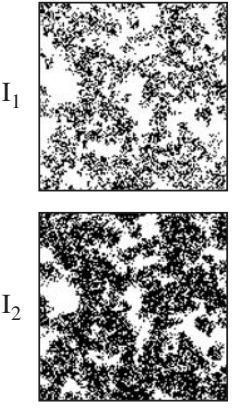

(a)
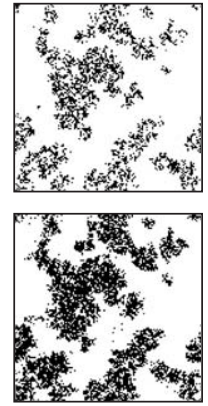

(b)

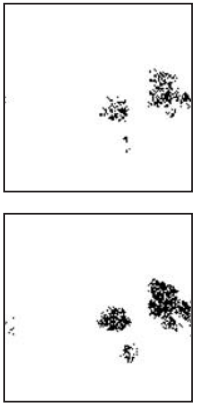

(c)

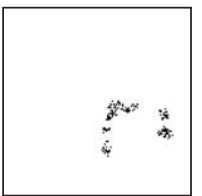

Fig. 15. Spatiotemporal dynamics for the asymmetric hypercycle with $\delta=1, k=1, \eta_{k}=1, \varepsilon=0.1945$ and $\eta_{\varepsilon}=\frac{1}{4}$. In this case the critical decay rate predicted by the mean field model is $\varepsilon_{c}=0.2$. The upper two trajectories display the time survival of both hypercycle replicators with $D=1$. On the contrary, both members become extinct with $D=0$. The inset shows (for the same parameter values as in the time series except for $\varepsilon \approx 0.249$ ) the percentage of hypercycle survival (in black) averaged over the final density values of 300 runs after $\tau=10^{4}$ generations, for (A) $D=0$ and (B) $D=0.95$. Note that with such a decay probability all the runs in (A) become extinct, while in (B) a $75 \%$ survive. The spatial patterns correspond to the extinction time trajectories (i.e. $D=0$ ) obtained at (a) 500, (b) 2000, (c) 8000 and (d) 11500 iterations. 
persistence scenario the second member of the hypercycle (the decay rate of which is lower because of the asymmetry) achieves higher stationary density values, while the first one undergoes a lower stationary density. As shown in the symmetric case, local diffusion plays a positive role in the maintenance of the network. Fig. 15 clearly shows the crucial effect of diffusion, $D$. Here a decay value very near the bifurcation point predicted by the mean field model is used. It is shown that the asymmetric hypercycle persists when diffusion probability is maximum. On the contrary, with $D=0$, both hypercycle members extinct (see Fig. 15 for details). Note that the spatial patterns show that, as in the symmetric case (see Fig. 11), the spatial distribution of both hypercycle species is quasi-identical, with a more dense pattern for the fittest, i.e. second, species of the network.

\section{Metapopulation hypercycle model}

In order to analytically define the previous stochastic model for the symmetric hypercycle we need to consider both replication and diffusion processes. This means to either explore a continuous, Turing-like approach or instead properly define the discrete rules of interaction. Although the introduction of diffusion might be a difficult task (Fontanari and Ferreira, 2002), here we show that such a model can be derived from a metapopulation approach (Bascompte and Solé, 1998; Hanski, 1999). Actually, as far as we know, this is the first time an explicit discrete model allows a mean field treatment of diffusion on a metapopulation context.

Our metapopulation model considers the symmetric twomember hypercycle in a scenario with an infinite number of compartments without explicitly taking into account local spatial interactions. Here, each compartment (patch in the metapopulation jargon) can be empty or occupied by one or by two replicators. The normalized frequency for such patches is indicated with the variables $x, y$ and $z$, respectively. Such a framework, as shown below, allows to properly define reactions and diffusion in a common setup. Hence, transitions between compartments are given by the next set of reactions:

$y \stackrel{e}{\longrightarrow} x$,

$z \stackrel{e^{2}}{\longrightarrow} x$

$z \stackrel{e}{\longrightarrow} y$

$z+x+s \stackrel{r}{\longrightarrow} z+y$,

$z+x \underset{D_{1}}{\stackrel{D_{-1}}{\leftrightarrows}} 2 y$

$z+y+s \stackrel{r}{\longrightarrow} 2 z$,

where $e$ and $r$ represent decay and replication rates, respectively; $D_{1}$ and $D_{-1}$ are the diffusion rates attending a bidirectional diffusion process (we assume that $D_{1}=$ $\left.D_{-1} \equiv D\right)$.

Here we also assume that $I_{1}$ and $I_{2}$ are kinetically equivalent but nevertheless distinguishable. Thus when replication or diffusion of replicators takes place, the moving replicator will occupy a compartment already occupied by one replicator $(y)$ with probability $\frac{1}{2}$, thus maintaining the condition that a replicator cannot fill a neighboring site containing the same type of molecule. Compartment dynamics, assuming the constraint $x+y+$ $z=1$, is given by the next set of ordinary differential equations:

$\dot{x}=e y+e^{2} z-r z x-D z x+\frac{1}{2} D y^{2}$,

$\dot{y}=r z x+2 D z x-e y-D y^{2}-\frac{1}{2} r z y+e z$,

$\dot{z}=\frac{1}{2} r z y-D z x-e^{2} z+\frac{1}{2} D y^{2}-e z$.

The dynamics of the three classes of patches is numerically obtained solving Eqs. (35)-(37) using the standard Euler method (with a constant time stepsize $\left.\delta t=10^{-3}\right)$. Here the stable and the extinction phases are also found, as well as the bifurcation scenario seen in Section 2. In Fig. 16 we represent, for three different initial conditions, the time evolution for all the compartments.
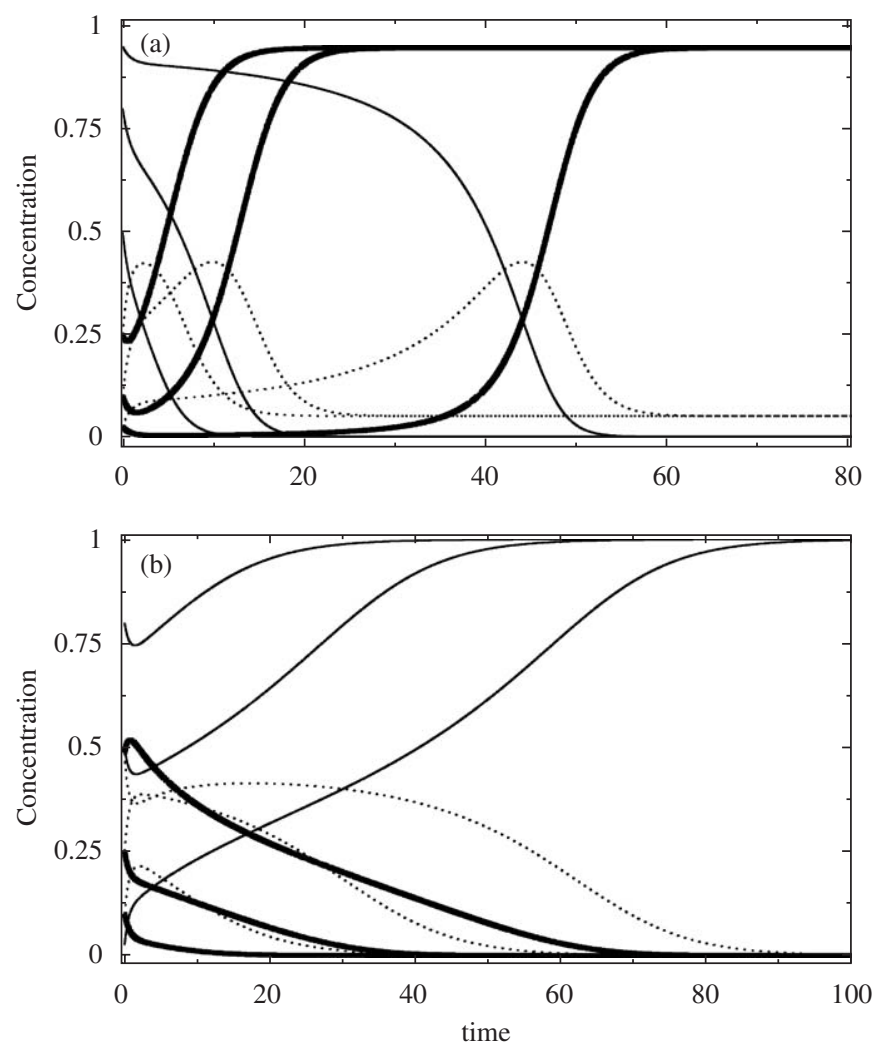

Fig. 16. Concentration time evolution for $x$ (solid thin line), $y$ (dotted line) and $z$ (solid thick line), from three different initial conditions. The trajectories characterize both phases of the two-member hypercycle: (a) the phase of stability with $e=0.05, r=1$ and $D=1$; and (b) the extinction phase, increasing the decay rate and decreasing replication, with $e=0.15, r=0.7$ and $D=1$. 
Specifically, two scenarios are considered: the phase were the hypercycle persists (Fig. 16a); and the extinction phase were the empty compartment concentration $x$ becomes maximum (Fig. 16b). Note that in the persistence phase the empty compartment, $x$, goes down to zero while the concentration for both $y$ and $z$ compartments reaches a non-trivial steady state. On the contrary, with the extinction phase, the empty compartment achieves the maximum concentration, i.e. $x=1$, while the concentration for the other two compartments becomes zero.

Phase portraits (Fig. 17) show the qualitative behavior involved in the persistence and in the extinction scenarios in this metapopulation approach. The fixed points, associated with persistence and extinction are given by two stable nodes (black circles), with a saddle-node in between. In Figs. 17a-c these three fixed points are homo- logous to those presented in Figs. 2a and b. A decrease in replication rate involves the approach among the coexistence fixed point and the saddle. As discussed in Section 2 (see Fig. 2), the motion and the collision of these fixed points cause a saddle-node bifurcation. After the bifurcation, any arbitrary initial condition flows toward the extinction attractor, which becomes globally stable.

In order to explore the parameters involved in this metapopulation approach we repeat the same analysis of Fig. 12, but now obtained from numerical solutions of Eqs. (35)-(37). Here it is also possible to see both phases, the one with persistence of replicators and the other with their extinction (see Fig. 18). It is shown that the extinction threshold obtained from this metapopulation approach is $e_{c} \approx 0.19$, which is close to the one characterized in the two-dimensional CA (remember $\varepsilon_{c} \approx 0.15$ ), both with
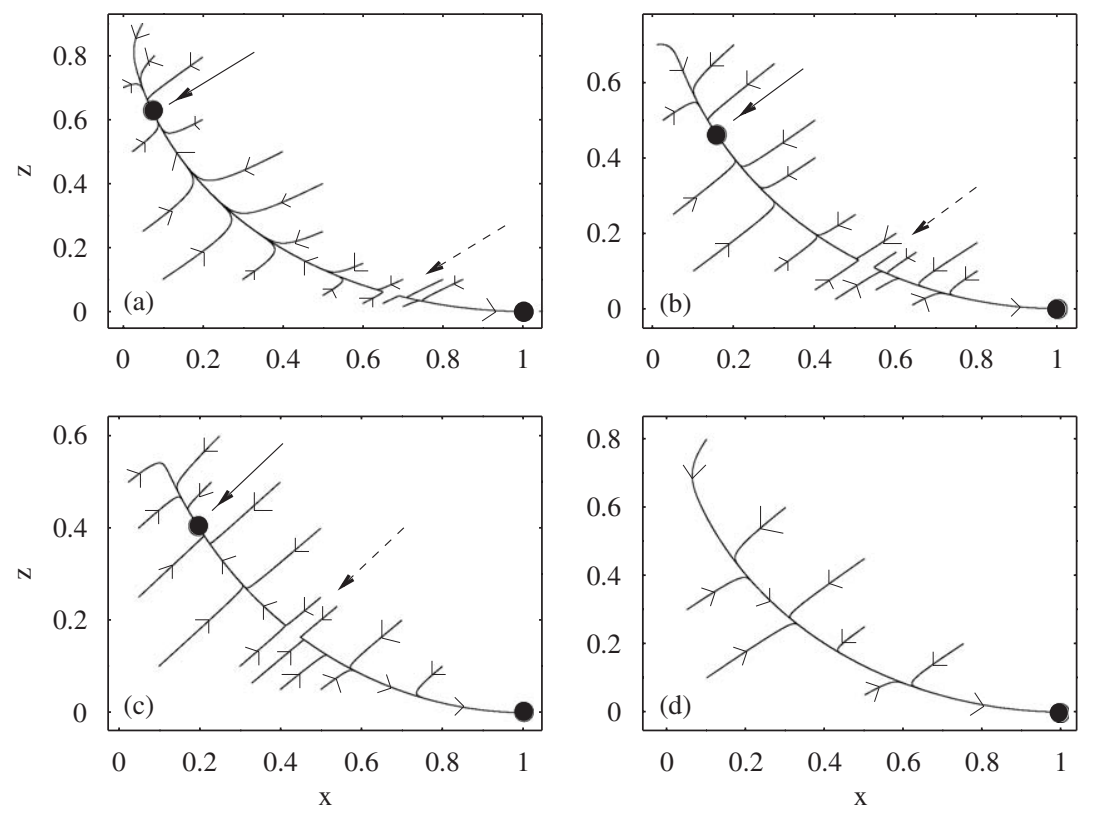

Fig. 17. Flow in phase space with $e=0.125, D=1$ and (a) $r=1$, (b) $r=0.75$, (c) $r=0.7$ and (d) $r=0.6$ obtained from numerical solutions of Eqs. (35)-(37), represented for the empty compartments $(x)$ and compartments with two replicators $(z)$. Black dots represent the stable fixed points: the coexistence node and the extinction attractor, respectively. At decreasing $r$ values the coexistence node (solid arrow) and the saddle-node (dashed arrow) approach each other. As shown in Section 2, the collision of both fixed points causes the saddle-node bifurcation and then all the phase space becomes the basin of attraction for the extinction attractor.

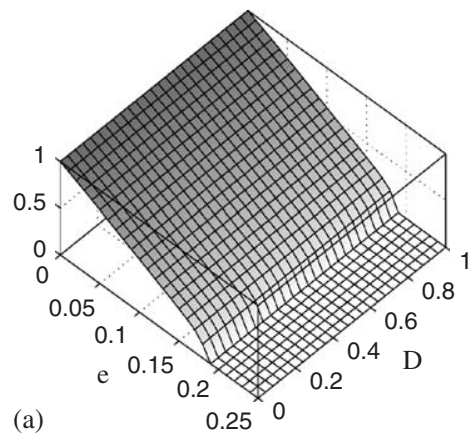

(b)
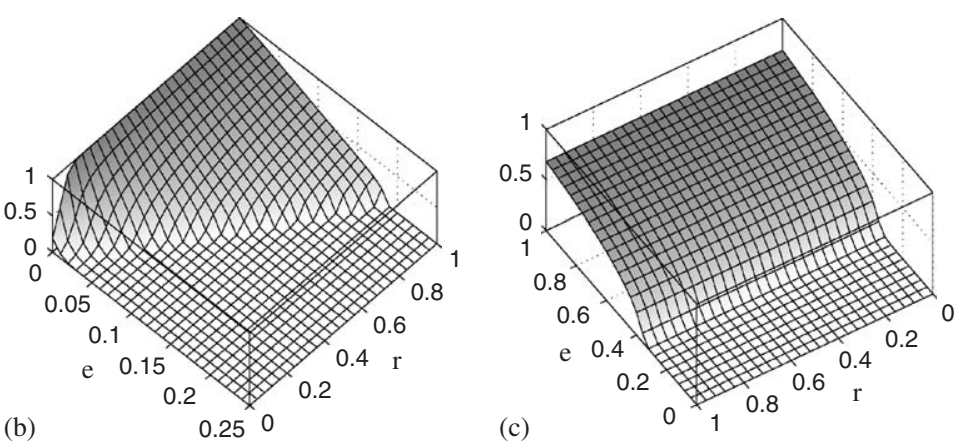

Fig. 18. Replicators density ( $z$-axis) obtained from Eqs. (35)-(37). In (a) decay-diffusion space with $r=1$; in (b) decay-reaction space with $D=1$; and in (c) reaction-diffusion space with $e=0.075$. These diagrams are consistent with those obtained from the spatially explicit model (see Fig. 12). 
$k=1, \delta=1$ and $D=1$. But the positive role of diffusion in the persistence of both replicators seen in the stochastic approaches is not reflected here (Figs. 18a and c), standing out the importance of local interactions in the explicit space.

\section{Discussion}

Space has played a key role in canalizing the evolution and selection of early replicators and is likely to be important in future, experimental synthesis of self-replicating molecules. Understanding the nature of transitions between different behavioral patterns is a relevant step in defining the potential scenarios where these replicators might successfully persist and evolve. In the present work we develop several models in order to analyse the dynamics of two-member hypercycles. The continuous, non-spatial dynamical system (Section 2) shows the existence of a critical decay rate below which three fixed points are shown to exist: two stable points and a saddle-node. The stable points are the attractors involved in both persistence (i) and extinction (ii) phases. Beyond $\varepsilon_{c}$ and after the coalescence of the coexistence node with the saddle-node in a saddlenode bifurcation that leaves a ghost in the phase plane, the only attractor of phase space is the extinction one (Figs. 2c,d), thus both hypercycle members asymptotically extinct (Fig. 3).

The mean field model used in Section 3.1 predicts the hyperbolic relation between diffusion associated with cross-catalytic replication (labeled $\delta$ ) and replication. The spatial analysis developed with the stochastic CAs (Section 3.2) allows to take into account the local nature of interactions. We also found extinction and coexistence phases, which are separated by sharp boundaries. Such models show that diffusion plays a positive role in the persistence of both replicators. In the two-dimensional CA the extinction phase is shown to be given by an absorbing state associated with a first-order phase transition (Figs. 9, 10 and 14), analogous to the saddle-node bifurcation scenarios shown in the mean field approaches. We have shown that the two-member hypercycle as a surfacebonded chemical network could increase its persistence in a medium favoring diffusion (Figs. 6-8, 10, 12, 14 and 15). Nevertheless, diffusion might play a negative role when considering extremely low densities of replicators as initial conditions (Fig. 13). Finally, a novel mean field metapopulation model considering diffusion $(D)$ of replicators is also considered in Section 4. The qualitative change between phases (i) and (ii) is shown and is also characterized by a saddle-node bifurcation (Fig. 17).

Our results confirm the relevance of spatial degrees of freedom as well as of the information encoded by the hypercycle in shaping the dynamics of hypercyclic organization. As it occurs with other metapopulation models, we also show the importance of dispersal (diffusion) as a key for success. Under similar conditions, the presence of diffusion allows the expansion through available space and the increasing opportunities for colonization increase the chances of maintaining a stable population. Future work should consider including in our description the presence of adaptive dynamics, thus allowing the offspring of the hypercycles to explore parameter space. In this context, the emergence of parasites, the role of stochasticity and the relevance of spatial structures in hypercyclic evolution should be analysed in more detail.

\section{Acknowledgments}

We want to thank the Complex Systems Lab staff as well as the reviewers of this work for useful comments and suggestions. This work has been supported by an EU PACE grant to JS within the 6th Framework Program under contract FP6-002035 (Programmable Artificial Cell Evolution) and by the Santa Fe Institute.

\section{References}

Bascompte, J., Solé, R.V., 1995. Rethinking complexity: modelling spatiotemporal dynamics in ecology. Trends Ecol. Evol. 10, 361-366.

Bascompte, J., Solé, R.V., 1998. Modeling Spatiotemporal Dynamics in Ecology. Springer, Berlin.

Boerlijst, M., 2000. In: Dieckmann, U., Law, R., Metz, J.A.J. (Eds.), The Geometry of Ecological Interactions. Simplifying Spatial Complexity. The Press Syndicate of the University of Cambridge, pp. 171-182.

Boerlijst, M.C., Hogeweg, P., 1991. Spiral wave structure in pre-biotic evolution: hypercycles stable against parasites. Physica D 48, 17-28.

Breyer, J., Ackermann, J., McCaskill, J., 1998. Evolving reaction-diffusion ecosystems with self-assembling structures in thin films. Artif. Life 4, 25-40.

Chacón, P., Moran, F., 1993. Estructuras espaciales en un sistema cerrado formado por especies autoreplicativas. An. Quím. 379-385.

Cronhjort, M.B., 1995. Ph.D. Thesis, Department of Physics. Royal Institute of Technology, Stockholm.

Cronhjort, M.B., 2000. In: Dieckmann, U., Law, R., Metz, J.A.J. (Eds.), The Geometry of Ecological Interactions. Simplifying Spatial Complexity. The Press Syndicate of the University of Cambridge, pp. $151-170$

Cronhjort, M.B., Blomberg, C., 1994. Hypercycles vs parasites in a twodimensional partial differential equations model. J. Theor. Biol. 169, 31-49.

Cronhjort, M.B., Blomberg, C., 1997. Cluster compartmentalization may provide resistance to parasites for catalytic networks. Physica D 101, 289-298.

Czarán, T., Szathmáry, E., 2000. In: Dieckmann, U., Law, R., Metz, J.A.J. (Eds.), The Geometry of Ecological Interactions. Simplifying Spatial Complexity. The Press Syndicate of the University of Cambridge, pp. 116-134.

Eigen, M., 2002. Error catastrophe and antiviral strategy (commentary). Proc. Natl. Acad. Sci. 99 (21), 13374-13376.

Eigen, M., Schuster, P., 1979. The Hypercycle. A Principle of Natural SelfOrganization. Springer, Berlin.

Eigen, M., Biebricher, C.K., Gebinoga, M., Gardiner, W.C., 1991. The hypercycle. Coupling of RNA and protein synthesis in the infection cycle of an RNA bacteriophage. Biochemistry 30 (46), 11005-11018.

Fontanari, J.F., Ferreira, C.P., 2002. Nonequilibrium phase transitions in a model for the origin of life. Phys. Rev. E 65, 021902-1,021902-10.

Füchslin, R.M., McCaskill, J.S., 2001. Evolutionary self-organization of cell-free genetic coding. Proc. Natl. Acad. Sci. 98 (16), 9185-9190.

García-Tejedor, A., Sanz-Nuño, J.C., Olarrea, J., de la Rubia, F.J., Montero, F., 1988. Influence of the hypercycle on the error threshold: a stochastic approach. J. Theor. Biol. 134, 431-443. 
Hanski, I., 1999. Metapopulation Ecology. Oxford Series in Ecology and Evolution. Oxford University Press, Oxford.

Ilachinsky, A., 2002. Cellular Automata. A Discrete Universe. World Scientific, Singapore.

Lee, D.H., Severin, K., Reza Ghadiri, M., 1997a. Autocatalyitic networks: the transition from molecular self-replication to molecular ecosystems. Curr. Opin. Chem. Biol. 1, 491-496.

Lee, D.H., Severin, K., Yokobayashi, Y., Reza Ghadiri, M., $1997 b$. Emergence of symbiosis in peptide self-replication through a hypercyclic network. Nature 390, 591-594.

Marro, J., Dickman, R., 1999. Nonequilibrium Phase Transitions in Lattice Models. Cambridge University Press, Cambridge.

McCaskill, J., 1997. Spatially resolved in vitro molecular ecology. Biophys. Chem. 66, 145-158.

Nuño, J.C., Andrade, M.A., Morán, F., Montero, F., 1993a. A model of an autocatalytic network formed by error-prone self-replicative species. Bull. Math. Biol. 55 (2), 385-415.

Nuño, J.C., Montero, F., de la Rubias, F.J., 1993b. Influence of external fluctuations on a hypercycle formed by two kinetically indistinguishable species. J. Theor. Biol. 165, 553-575.

Rowe, G.W., 1997. Theoretical Models in Biology: The Origins of Life, the Immune System and the Brain. Oxford University Press, Oxford.

Scheuring, I., Czárán, T., Szabó, P., Károlyi, G., Toroczkai, Z., 2002. Spatial models of prebiotic evolution: soup before pizza? Origins Life Evol. Biosphere 33, 319-355.
Smith, J.M., Szathmáry, E., 2001. The Major Transitions in Evolution. Oxford University Press, Oxford.

Solé, R.V., Bascompte, J., 2006. Self-organization in Complex Ecosystems. Monographs in Population Biology. Princeton Uinversity Press, Princeton.

Solé, R.V., Goodwin, B., 2000. Signs of Life: How Complexity Pervades Biology. Basic Books, New York.

Stadler, B.M.R., Stadler, P.F., 2003. Molecular Replicator Dynamics. Adv. Complex Syst. 6, 47-77.

Strogatz, S.H., 2000. Nonlinear Dynamics and Chaos with Applications to Physics, Biology, Chemistry, and Engineering. Westview Press, Boulder, CO.

Strogatz, S.H., Westervelt, R.M., 1989. Predicted power laws for delayed switching of charge density waves. Phys. Rev. B 40 (15), 10501-10508.

Swetina, J., Schuster, P., 1982. Self-replication with errors. A model for polynucleotide replication. Biophys. Chem. 16, 329.

Szabó, P., Scheuring, I., Czárán, T., Szathmáry, E., 2002. In silico simulations reveal that replicators with limited dispersal evolve towards higher efficiency and fidelity. Nature 420, 340-343.

Szathmáry, E., Maynard Smith, J., 1997. From replicators to reproducers: the first major transitions leading to life. J. Theor. Biol. 169, 555-571.

Wächtershäuser, G., 1988. Before enzymes and templates: theory of surface metabolism. Microbiol. Rev. 52 (4), 452-484. 